\title{
A mechanism to derive multi-power law functions: an application in the econophysics framework.
}

\author{
A.M. Scarfone \\ Istituto Nazionale di Fisica della Materia (CNR-INFM) and Physics Department \\ Unitá del Politecnico di Torino, Corso Duca degli Abruzzi 24, \\ I-10129 Torino, Italy
}

\begin{abstract}
It is generally recognized that economical systems, and more in general complex systems, are characterized by power law distributions. Sometime, these distributions show a changing of the slope in the tail so that, more appropriately, they show a multi-power law behavior. We present a method to derive analytically a two-power law distribution starting from a single power law function recently obtained, in the frameworks of the generalized statistical mechanics based on the Sharma-TanejaMittal information measure. In order to test the method, we fit the cumulative distribution of personal income and gross domestic production of several countries, obtaining a good agreement for a wide range of data.
\end{abstract}

Key words: Two-power law distribution, Sharma-Taneja-Mittal information measure, distribution of personal income and gross domestic production.

PACS: 02.50.-r, 89.65.Gh, 89.75.Da

\section{Introduction}

Free-scale behavior in the economical systems have been observed since 19th century, when Pareto noticed that the cumulative distribution of the personal income $P(x)=\int_{x}^{\infty} p(y) d y$ of several countries behaves like a power law function. Afterwards, Gibrat clarified that such a power law behavior holds only for the high income region, whilst in the low-middle income region, which includes almost the whole body of data, the curve is well fitted by a log-normal distribution.

Actually, the problem concerning the real profile showed by the function $P(x)$ in the whole range of the accessible data is still an open question. In particular, 
it has been suggested [1] that deformed exponential functions derived recently in the field of the generalized statistical mechanics, can be fruitfully employed to modeling analytically the cumulative distribution $P(x)$ for a wide range of the income values.

Notwithstanding, the recent analysis based on a huge quantity of data nowadays accessible, shown that sometime the crossover among the low-middle region (the log-normal region) and the high region in the upper tail of the distribution (the Pareto region, with a power law behavior $P(x) \sim x^{-s}$, where $s$ is a positive constants quite generally $1 \leq s \leq 2$ ), does not occur smoothly, giving origin to knee or ankle effects (see for instance [2]). Moreover, in some cases, it has been observed a deviation from the Pareto behavior in the highest region, which can originate a new power law behavior $P(x) \sim x^{-\tilde{s}}$ with a different slope $\tilde{s} \neq s$.

The complicate profile in the shape of $P(x)$ cannot be accounted for by a generalized exponential with a single power law behavior. This open the questions: how can we describe the shape observed in $P(x)$ with an analytically simple function?

In the present contribution, we introduce a mechanism which permits to generate multi-power law functions by employing deformed exponentials and logarithms with a single power law asymptotic behavior.

Notice that, two-power law behavior have been observed in various economical systems like, for instance, in the cumulative distribution of the personal income [3], in the cumulative distribution of the land price [4] or in the returns of many market indexes [5].

On a general ground, two-power law behavior have been observed in different physics fields as well as in biological, geological and social sciences. Among the many, we quote the dielectric relaxation [6], the re-association in folder proteins [7], and others [8].

It is worthy to remark that there have been proposed different methods in literature [7,9,10] to produce generalized distributions with a double-power law behavior which differ from the one advanced in the following.

\section{Deformed logarithms and exponentials}

Generalized exponential functions $\mathcal{E}(x)$, interpolating between the standard exponential $\exp (x)$ for $x \ll 1$ and the power law $x^{-s}$ for $x \gg 1$, arise naturally in the study of thermostatistic proprieties of complex systems which show freescale feature. In [11, it has been postulated a very general expression for the entropy of such a system

$$
S(p)=-\int p(x) \Lambda(p(x)) d x
$$


(in the unity of Boltzmann constant $k_{\mathrm{B}}=1$ ), where $\Lambda(x)$ plays the role of a generalized logarithm, the inverse function of $\mathcal{E}(x)$. By requiring that the distribution, derivable through a variational problem, assumes the form

$$
p(x)=\mathcal{E}\left(-\sum_{j=1}^{M} \beta_{j}|x|^{\mu_{j}}\right),
$$

which mimics the well-known Boltzmann-Gibbs distribution, the following functional equation has been obtained

$$
\frac{d}{d x}[x \Lambda(x)]=\lambda \Lambda\left(\frac{x}{\alpha}\right) .
$$

Here, $\alpha$ and $\lambda$ are constants given by

$$
\alpha=\left|\frac{1+r-\kappa}{1+r+\kappa}\right|^{1 / 2 \kappa}, \quad \lambda=\frac{|1+r-\kappa|^{(r+\kappa) / 2 \kappa}}{|1+r+\kappa|^{(r-\kappa) / 2 \kappa}} .
$$

The quantities $\beta_{j}$ in Eq. (2.2) play the role of Lagrange multipliers associated to the $M$ constraints $\int|x|^{\mu_{j}} p(x) d x=\mathcal{O}_{j}$ which represent the $\mu_{j}$-th momenta of $x$. Typically, the constants $\mu_{j}$ are integers (for instance, $\mu_{1}=0$ gives the normalization $\int p(x) d x=\mathcal{O}_{1}, \mu_{2}=1$ is the mean value $\langle x\rangle=\mathcal{O}_{2}$, and so on) but for sake of generality we assume $\mu_{j} \in \mathbb{R}$.

The most general solution of Eq. (2.3), accounting for the boundary conditions $\Lambda(1)=0$ and $\left.(d / d x) \Lambda(x)\right|_{x=1}=1$, derived from certain physically and mathematically justified assumptions, is given by

$$
\Lambda(x) \equiv \ln _{\{\kappa, r\}}(x)=x^{r} \frac{x^{\kappa}-x^{-\kappa}}{2 \kappa},
$$

which recover the standard logarithm in the $(\kappa, r) \rightarrow(0,0)$ limit.

By requiring that $\ln _{\{\kappa, r\}}(x)$ is a continuous, monotonic, normalizable, concave and increasing function for $x \in(0,+\infty)$, we obtain the restrictions $-|\kappa| \leq r \leq|\kappa|$, if $0 \leq|\kappa|<1 / 2$ and $|\kappa|-1 \leq r \leq 1-|\kappa|$, if $1 / 2 \leq|\kappa|<1$. Notwithstanding, for particular applications some of the above mathematical requirements can be relaxed permitting less restrictive conditions for the deformation parameters. For instance, in certain practical situations one is welling with a normalization in a finite interval $x \in\left(0, x_{\max }\right)[12$ and we can discard the condition $|\kappa|<1$.

In the following, we require only that Eq. (2.5) be a monotonic function, so that its inverse function, the generalized exponential $\exp _{\{\kappa, r\}}(x)$, certainly exists. This is accomplished by requiring only that $-|\kappa|<r<|\kappa|$.

From Eq. (2.5) we obtain that $\ln _{\{\kappa, r\}}(x) \rightarrow x^{r+|\kappa|} /|2 \kappa|$ for $x \rightarrow+\infty$ and 
$\ln _{\{\kappa, r\}}(x) \rightarrow-x^{r-|\kappa|} /|2 \kappa|$ for $x \rightarrow 0$, whilst $\ln _{\{\kappa, r\}}(x) \rightarrow(x-1)$ for $|x-1| \ll 1$. In the same way, we have that $\exp _{\{\kappa, r\}}(x) \rightarrow|2 \kappa x|^{1 /(r \pm|\kappa|)}$ for $x \rightarrow \pm \infty$, whilst $\exp _{\{\kappa, r\}}(x) \rightarrow 1+x$ for $x \rightarrow 0$. Thus, the deformed exponential $\exp _{\{\kappa, r\}}(x)$ interpolates with continuity between the standard $\operatorname{exponential~} \exp (x) \simeq 1+x$, for $x \rightarrow 0$, and the power law $|x|^{-s}$ with slope $s=-1 /(r \pm|\kappa|)$, for $x \rightarrow \pm \infty$. Finally, accounting for the solution (2.5), the entropy (2.1) assumes the form

$$
S_{\kappa, r}(p)=-\int p(x) \ln _{\{\kappa, r\}}(p(x)) d x
$$

which recovers, in the limit $(\kappa, r) \rightarrow(0,0)$, the Shannon-Boltzmann-Gibbs entropy $S=-\int p(x) \ln p(x) d x$. This entropic form, introduced previously in literature in [13,14,15, is known as the Sharma-Taneja-Mittal information measure and has been applied recently in the formulation of a possible thermostatistics theory [16]17].

\section{Two-power law function}

Endowed with the deformed logarithm $\ln _{\{\kappa, r\}}(x)$ and the deformed exponential $\exp _{\{\kappa, r\}}(x)$ we can construct the quantity

$$
\Pi_{\sigma_{1}}(x)=\exp _{\left\{\kappa_{1}, r_{1}\right\}}\left(a_{1} \ln _{\left\{\kappa_{1}, r_{1}\right\}}(x)\right)
$$

where $\sigma_{1}$ denotes the set of parameters $\sigma_{1} \equiv\left(\kappa_{1}, r_{1}, a_{1}\right)$, with $a_{1} \geq 1$. The function (3.1) is therefore employed in the following construction

$$
f(x)=\Pi_{\sigma_{1}} \circ \exp _{\left\{\kappa_{2}, r_{2}\right\}}(x) \equiv \Pi_{\sigma_{1}}\left(\exp _{\left\{\kappa_{2}, r_{2}\right\}}(-x)\right) .
$$

We observe that, for $a_{1}=1$ expression (3.2) reduces to $\exp _{\left\{\kappa_{2}, r_{2}\right\}}(-x)$, for $\left(\kappa_{1}, r_{1}\right) \rightarrow(0,0)$ we obtain $\left[\exp _{\left\{\kappa_{2}, r_{2}\right\}}(-x)\right]^{a_{1}}$, whilst for $\left(\kappa_{1}, r_{1}\right) \stackrel{\left\{\kappa_{2}, r_{2}\right\}}{=}\left(\kappa_{2}, r_{2}\right)$ we obtain $\exp _{\left\{\kappa_{1}, r_{1}\right\}}\left(-a_{1} x\right)$.

Accounting for the asymptotic behavior of the deformed exponential and logarithm we can distinguish three regions in the range $x>0$ of $f(x)$. A first region, for $a_{1} x \ll 1$, characterized by the linear behavior

$$
f(x) \sim 1-a_{1} x
$$

like the exponential $\exp \left(-a_{1} x\right)$ does for $x \rightarrow 0$. A second intermediate region, for $x \ll 1 \ll a_{1} x$, where $f(x)$ is characterized by the power law behavior

$$
f(x) \sim x^{-s_{1}}
$$


with slope $s_{1}=1 /\left(\left|\kappa_{1}\right|-r_{1}\right)$.

Finally, for $x \gg 1$ we obtain the asymptotic power law behavior

$$
f(x) \sim x^{-s_{2}}
$$

whose slope is now $s_{2}=1 /\left(\left|\kappa_{2}\right|-r_{2}\right)$.

Thus, $f(x)$ behaves like a power law function both in the middle and in the far region of $x>0$ with slopes $s_{1}$ and $s_{2}$, respectively. In this sense, we call Eq. (3.2) a two-power law function.

Form the above analysis, we easily realize that the constant $a_{1}$, introduced in the definition of $\Pi_{\sigma_{1}}(x)$, gives approximatively the width of the intermediate region having slope $s_{1}$.

As an example, let us specialize Eq. (3.2) to the case $r=0$. In this situation,

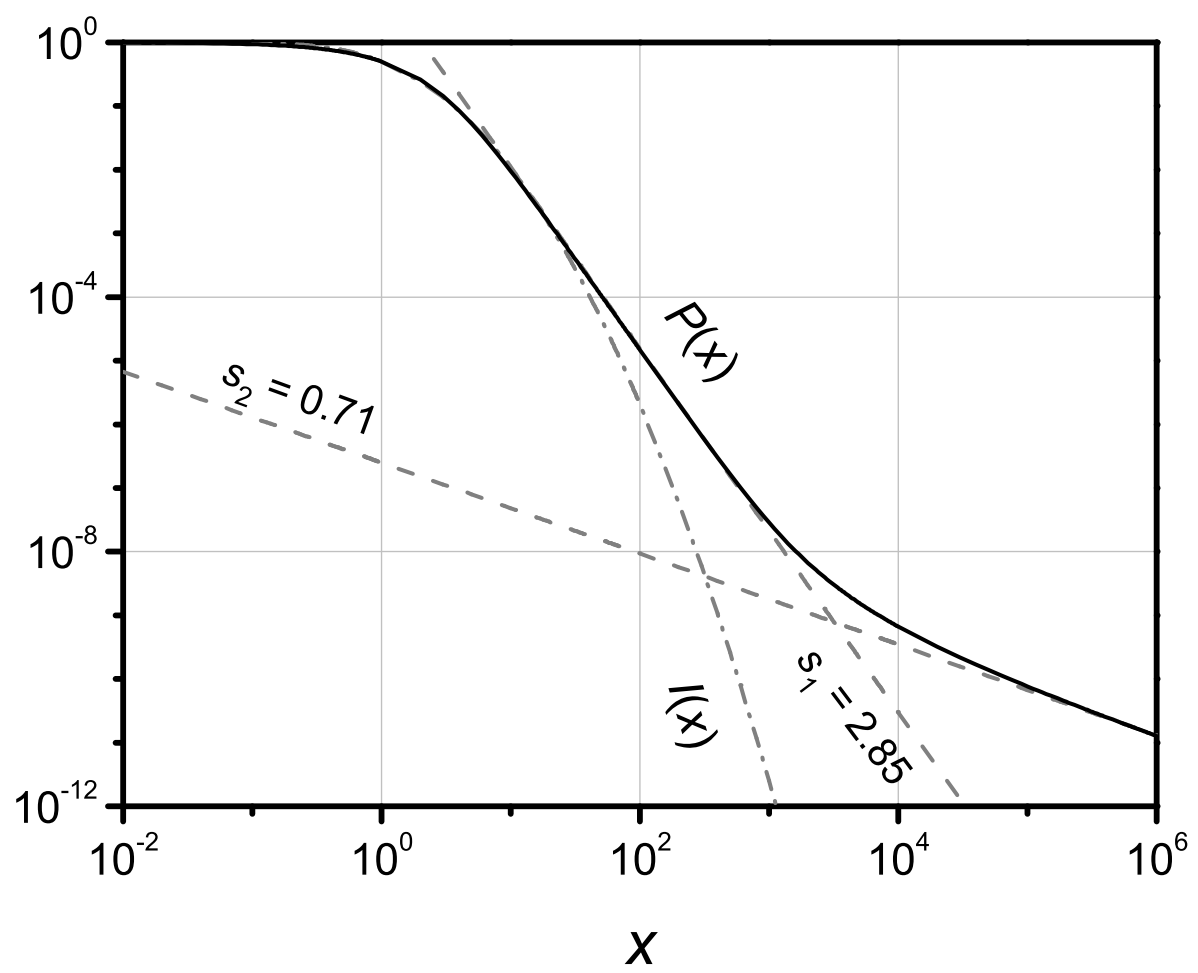

Fig. 1. Log-log plot of the two-power law function (3.2) (solid line). The dot-dashed line is the cumulative integral of the log-normal function. The dashed lines denote the asymptotic extension of the function $P(x)$ in the two-power law regions.

the generalized exponential and logarithm assume, respectively, the expression 
[20]

$$
\exp _{\{\kappa\}}(x)=\left(\kappa x+\sqrt{1+\kappa^{2} x^{2}}\right)^{1 / \kappa},
$$

and

$$
\ln _{\{\kappa\}}(x)=\frac{x^{\kappa}-x^{-\kappa}}{2 \kappa} .
$$

In figure 1 , we plot the function

$$
P(x)=\exp _{\left\{\kappa_{1}\right\}}\left(a_{1} \ln _{\left\{\kappa_{1}\right\}}\left(\exp _{\left\{\kappa_{2}\right\}}(-x)\right)\right),
$$

for the values $\kappa_{1}=0.35, \kappa_{2}=1.4$ and $a_{1}=10^{3}$. In the same graphic, the dot-dashed line depicts the cumulative integral $I(x)=\int_{x}^{\infty} p(y) d y$ of the lognormal distribution

$$
p(x)=\frac{1}{(2 \pi)^{1 / 2} x} \exp \left(-\frac{1}{2} \ln ^{2} x\right) .
$$

The dashed lines represent the asymptotic prolongation of the power law behavior of $P(x)$ whose slopes are given, respectively, by $s_{1}=1 / k_{1}=2.85$ and $s_{2}=1 / k_{2}=0.71$. We observe a good agreement between the functions $I(x)$ and $P(x)$ only in the low region of $x$.

\section{Application to econophysics}

In the following, we employ the function derived in the previous Section 3 to fit some distributions data obtained in the economy framework.

We pose $P(x)=f\left(-\beta|x|^{\mu}\right)$, with $\beta$ and $\mu$ fitting parameters, the cumulative distribution representing the probability of finding a value $X$ equal to, or greater than $x$.

In figure 2, we present the results of the fit (in log-log scale) for the data of the inverse cumulative distribution of the personal income of Japan (1975) obtained in [18] and USA (2000) obtained in [3], as well as, the data of the inverse cumulative distribution of the gross domestic production of Brazil (1996) and Germany (1998) obtained in [3].

In every graphic, we report the dashed lines representing the asymptotic behavior of $P(x)$ in the two power law regions with slope given by $s_{1}=\mu / k_{1}$ and $s_{2}=\mu / k_{2}$, respectively.

The data fit are reported in table 1 . 

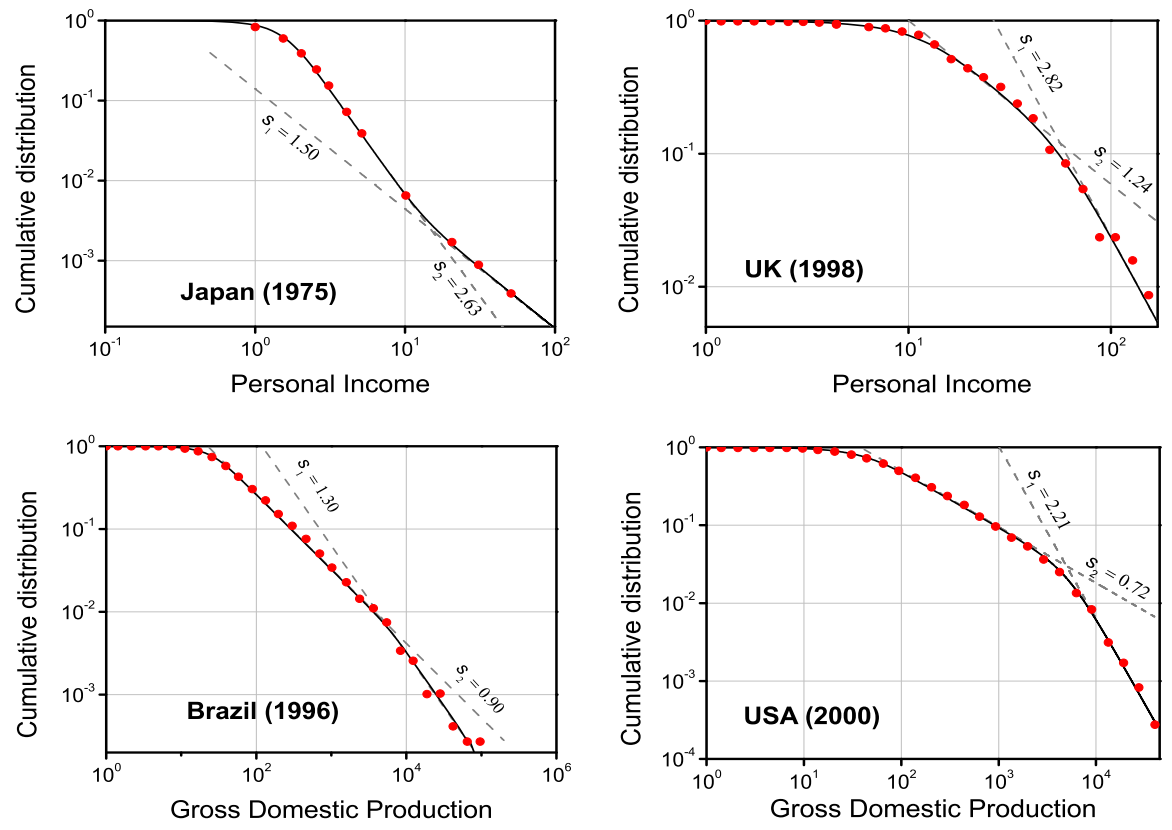

Fig. 2. Log-log plot of personal income distribution for Japan (1975) [18] and USA (2000) [3] and gross domestic production distribution for Brazil (1996) and Germany (1998) [3]. The solid line represents the fit obtained with the two-power law function (3.8). The straight dashed lines are plotted for convenience to indicate the asymptotic power-law prolongation.

Table 1.

Parameters for the cumulative distribution $P(x)$.

\begin{tabular}{lccccc}
\hline Country & $\kappa_{1}$ & $\kappa_{2}$ & $a_{1}$ & $\mu$ & $\beta$ \\
\hline Japan (1975) & 1.14 & 2.00 & 390 & 3.00 & $3.5 \cdot 10^{-4}$ \\
UK (1998) & 1.70 & 0.75 & 8 & 2.12 & $2.5 \cdot 10^{-4}$ \\
Brazil (1996) & 2.20 & 1.53 & $2 \cdot 10^{4}$ & 1.99 & $2.3 \cdot 10^{-8}$ \\
USA (2000) & 2.00 & 0.65 & 231 & 1.44 & $6.0 \cdot 10^{-6}$ \\
\hline
\end{tabular}

The crossover between the first and the second power law region, causing a reduction of the slope, with $s_{2}<s_{1}$ (UK, Brazil and USA), is named kink effect [3]. Similarly, the crossover between the first and the second power law region causing an increase of the slope, with $s_{2}>s_{1}$ (Japan), is named ankle effect. 


\section{Generalization}

Let us briefly discuss the generalization of the method introduced in Section 3 in order to generate functions with more than two power law behavior. This can be accomplished starting from the building block function

$$
\Pi_{\left\{\sigma_{i}\right\}}(x)=\exp _{\left\{\kappa_{i}, r_{i}\right\}}\left(a_{i} \ln _{\left\{\kappa_{i}, r_{i}\right\}}(x)\right),
$$

and introducing the quantity

$$
\Pi_{\{\vec{\sigma}\}}(x)=\Pi_{\left\{\sigma_{1}\right\}} \circ \Pi_{\left\{\sigma_{2}\right\}} \circ \ldots \circ \Pi_{\left\{\sigma_{n-1}\right\}}(x),
$$

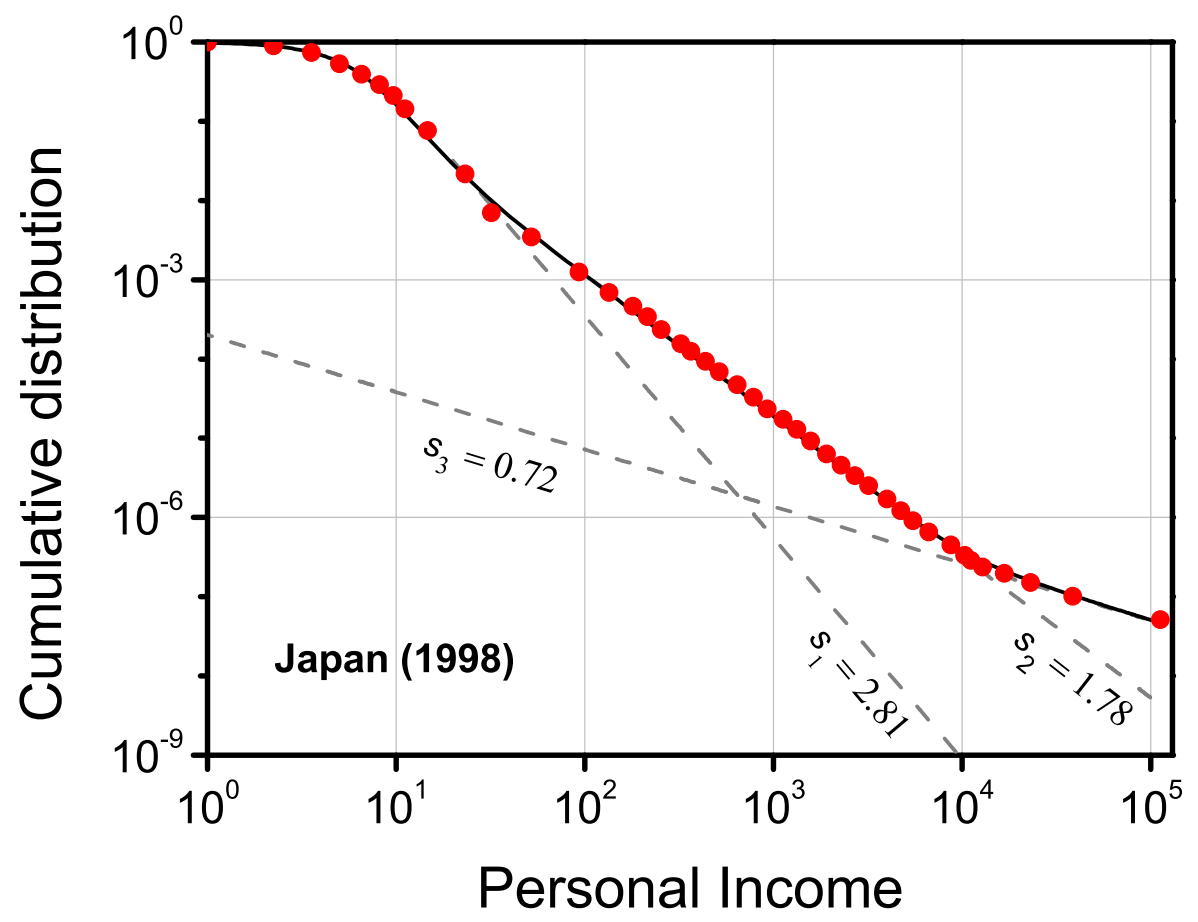

Fig. 3. Log-log plot of the cumulative distribution of Japan for the year 1988. It is observed a deviation from the Pareto behavior in the highes income region $\left(x>10^{4}\right)$.

where $\vec{\sigma} \equiv\left(\sigma_{1}, \sigma_{2}, \ldots, \sigma_{n-1}\right)$ is a $(n-1)$-vector whose $i$ th entry $\sigma_{i}=\left(\kappa_{i}, r_{i}, a_{i}\right)$ contains the relevant informations about the slope and the width of the $i$ th power law region. It is easy to verify that the function

$$
f(x)=\Pi_{\{\vec{\sigma}\}} \circ \exp _{\left\{\kappa_{n}, r_{n}\right\}}(-x),
$$


exhibits a $n$-power law behavior.

In figure 3, we report the fit of the 1998 Japanese income data obtained in [19] by employing the function

$$
P(x)=\Pi_{\{\vec{\sigma}\}} \circ \exp _{\left\{\kappa_{3}\right\}}\left(-\beta x^{\mu}\right),
$$

derived from Eq. (5.3) for $n=3$ and $r_{i}=0$. The fitting data are $\kappa_{1}=$ $0.71, \kappa_{2}=1.12, \kappa_{3}=2.77, a_{1}=10, a_{2}=4 \cdot 10^{5}, \beta=6.00 \cdot 10^{-9}$ and $\mu=2.00$.

\section{Conclusions}

We have derived a simple method which permits to generate functions with a multi-power law behavior starting the deformed logarithm $\ln _{\{\kappa, r\}}(x)$ and the deformed exponential $\exp _{\{\kappa, r\}}(x)$, recently derived in [11], which exhibit a single power law profile. An explicit two-power law function has been constructed starting from the $\kappa$-exponential and its inverse, the $\kappa$-logarithm. We have employed this function to fit the inverse cumulative distribution of the personal income and of the gross domestic production of several countries, showing a good agreement among the analytical and the empirical data for a wide range of values.

\section{References}

[1] F. Clementi and M. Gallegati, Econophysics of Wealth Distributions, Ed.s A. Chatterjee, S. Yarlagadda and B.K. Chakrabarti, (Milan: Springer-Verlag, Italia 2005).

[2] A.C. Silva and V.M. Yakovenko, Erophysics Lett. 69, 304 (2005).

[3] E.P. Borges, Physica A 334, 255 (2004).

[4] A. Ishikawa, Physica A 371, 525 (2006).

[5] P. Gopikrishnan, V. Plerou, L.A. Plerou, M. Meyer, and H.E. Stanley, Phys. Rev. E 60, 5305 (1999).

[6] K. Weron and A. Jurlewicz, J. Phys. A 26, 395 (1993).

[7] C. Tsallis, G. Bemski, and R.S. Mendes, Phys. Lett. A 257, 93 (1999).

[8] M. Montemurro, Physica A 300, 567 (2001).

[9] M. Czachor and J. Naudts, Phys. Lett. A 298, 369 (2002). 
[10] W. Li, Q.A. Wang, L. Nivanen, and A. le Méhauté, Eur. Phys. J. B 48, 95 (2005).

[11] G. Kaniadakis, M. Lissia, and A.M. Scarfone, Physica A 340, 41 (2004); Phys. Rev. E 71, 046128 (2005).

[12] M.E.J. Newman, Contemp. Phys. 46, 323 (2005).

[13] B.D. Sharma and I.J. Taneja, Metrika 22, 205 (1975).

[14] B.D. Sharma and D.P. Mittal, J. Math. Sci. 10, 28 (1975).

[15] D.P. Mittal, Metrika, 22, 35 (1975).

[16] A.M. Scarfone and T. Wada, Phys. Rev. E 72, 026123 (2005).

[17] A.M. Scarfone, Physica A 365, 63 (2006).

[18] W. Souma, Physics of personal Income, arXiv:cond-mat/0202388.

[19] W. Souma, Fractals 9, 463 (2001).

[20] G. Kaniadakis, Phys. Rev. E 66, 056125 (2002); Phys. Rev. E 72, 036108 (2005). 